J Phys Chem Lett. 2016 October 06; 7(19): 3798-3803. doi:10.1021/acs.jpclett.6b01722.

\title{
Reconciling Intermediates in Mechanical Unfolding Experiments With Two-State Protein Folding in Bulk
}

\author{
David de Sancho ${ }^{\dagger,}$ and Robert B. Best ${ }^{\ddagger}$ \\ †CIC nanoGUNE, 20018 Donostia-San Sebastian, Spain \\ FLaboratory of Chemical Physics, National Institute of Diabetes and Digestive and Kidney \\ Diseases, National Institutes of Health, Bethesda, MD 20892-0520 \\ IIKERBASQUE, Basque Foundation for Science, María Díaz de Haro 3, 48013 Bilbao, Spain
}

\begin{abstract}
Most experimentally well-characterized single domain proteins of less than 100 residues have been found to be two-state folders. That is, only two distinct populations can explain both equilibrium and kinetic measurements. Results from single molecule force spectroscopy, where a protein is unfolded by applying a mechanical pulling force to its ends, have largely confirmed this description for proteins found to be two state in ensemble experiments. Recently, however, stable intermediates have been reported in mechanical unfolding experiments on a cold-shock protein previously found to be a prototypical two-state folder. Here, we tackle this discrepancy using free energy landscapes and Markov state models derived from coarse-grained molecular simulations. We show that protein folding intermediates can be selectively stabilized by the pulling force and that the populations of these intermediates vary in a force-dependent manner. Our model qualitatively captures the experimental results and suggests a possible origin of the apparent discrepancy.
\end{abstract}

\section{TOC image}

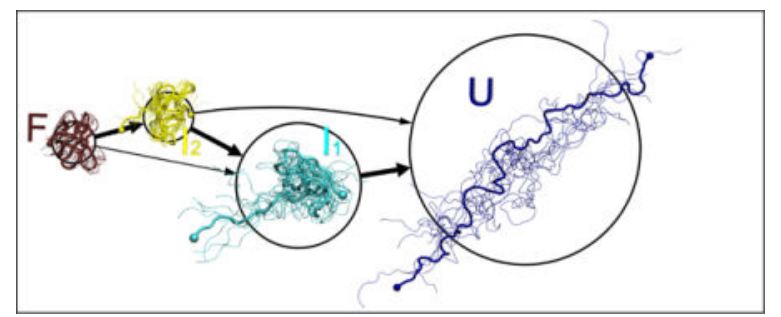

The development of single molecule technologies over the past two decades has allowed the observation of details of protein free energy landscapes that had been inaccessible to ensemble experiments. ${ }^{1}$ In single molecule force spectroscopy experiments a pulling force is applied directly to the termini of the protein, resulting in unfolding. Evidence from both

Correspondence to: David de Sancho; Robert B. Best.

Supporting Information Available: Detailed description of the methods and additional figures. This material is available free of charge via the Internet at http://pubs.acs.org/. 
experiment and computer simulations indicates that the folding mechanism may change as a function of force, including the population of intermediates ${ }^{2-6}$ as well as changes in folding transition state (via $\phi$-value analysis in experiment) ${ }^{6-12}$ However, for most proteins which have been shown to be two-state ${ }^{13}$ from bulk experiments in the absence of force, only a folded and an extended unfolded state become observable in the pulling experiments, confirming the existing description. In other cases, in which more than two stable states were identified in force experiments, the unfolding mechanism was generally consistent with previous equilibrium and kinetic measurements. ${ }^{14}$ The recent increase in stability of the instruments and the use of different measurement modes such as force clamp ${ }^{15,16}$ and force ramp ${ }^{17}$ have further helped to reveal discrepancies between mechanical and ensemble unfolding mechanisms. ${ }^{18,19}$

Here we focus in the thermostable protein CspB from Thermotoga maritima, Csp Tm (see Fig. 1a). For this system, equilibrium fluorescence measurements and stopped flow kinetic experiments have universally indicated two-state folding, ${ }^{20}$ a finding later confirmed at the single molecule level using FRET. ${ }^{21,22}$ AFM experiments in the constant speed mode also fit with a two-state description. ${ }^{23}$ However, recent work has clearly identified multiple metastable states which are populated when pulling the protein from its ends in the constant force mode. ${ }^{24}$ Intermediates in single molecule force experiments had previously been identified, perhaps the best known example being Titin I27. ${ }^{2-5}$ What is remarkable in this case is the longevity (milliseconds in the presence of a pulling force) and heterogeneity of the intermediates, considering the small size of Csp $T m$ (66 residues), and the fact that no intermediates were resolved in ensemble experiments. To tackle this problem we run molecular simulations of a coarse-grained topology based model of Csp Tm and derive a force-dependent Markov state model (MSM) that captures the kinetics of the system. In accord with experiment, we find that while in the absence of force the system is a two-state folder by thermodynamic and kinetic criteria, in the presence of a constant biasing force, certain intermediate species are stabilized. The kinetic model we present recapitulates the shift from two-state to multi-state folding upon application of force recently discovered experimentally.

Using the experimental NMR structure of the protein ${ }^{25}$ as a starting point (see Fig. 1a) we have used well established methods to build a topology-based coarse-grained (i.e. Gō) model $^{26}$ (see SI for details). Such models, in which only native contacts are considered attractive, are justified in the context of a funneled folding energy landscape ${ }^{27}$ First we check whether this simple model is able to recapitulate the two-state description in the absence of force. We have run simulations without a pulling force at multiple temperatures and combined them using WHAM. ${ }^{28}$ The projection on the folding coordinate $Q$ (fraction of native contacts) ${ }^{29}$ of one of these trajectories, corresponding to a temperature where we observe reversible folding ( $T=310 \mathrm{~K}$ ) shows how the protein inter-converts in sharp, cooperative, transitions between the folded and unfolded states (Fig. 1b). The calculated heat capacity thermogram and potential of mean force (PMF) on $Q$ both show unequivocal twostate features (see Fig. 1c-d). The former presents a single, well defined, narrow peak as a function of the temperature, with the simulation midpoint temperature located around $T_{m} \simeq$ $315 \mathrm{~K}$ (Fig. 1c). This is low compared to the experimental value (Thermotoga maritima grows up to $90^{\circ} \mathrm{C}^{20}$ ) but enough to yield a folding barrier in native conditions. In the same 
way, from the PMF we can also diagnose two-stateness for the Csp Tm model: there are two well defined wells, albeit with a small shoulder at an intermediate value of the fraction of native contacts $(Q=0.6)$. The two-state description is consistent with the simulation work carried out with a similar native-centric coarse-grained model where the denaturation effects from chemicals, instead of temperature, are explicitly accounted for. ${ }^{30}$

To analyze the effects of an external force in the system we have run multiple simulations pulling from the protein termini at different forces (see Table S1). The situation that we encounter is quite different from that at zero force. In Fig. 2a we show time series data for the projection on the pulling coordinate ( $d$, the end to end distance) for simulations carried out at different pulling forces and $300 \mathrm{~K}$. While at zero force the protein remains in the folded state but for transient excursions to higher distance conformations (i.e. the shoulder in the PMF from Fig. 1d), at non-zero forces intermediate species are clearly stabilized in the 2-6 nm range. At forces between 10 and $15 \mathrm{pN}$ we see an additional shorter-lived intermediate at $\sim 7-8 \mathrm{~nm}$ (see traces in Fig. S1). Our projected data resembles some of the experimental traces obtained with the AFM at constant force. ${ }^{24}$ In the experiments, up to 5 different intermediates could be observed, although in the majority of individual pulls the number of intermediates was either zero or just one. Also, considering an approximate value of $L_{\mathrm{O}} \simeq 1.3 \AA$ for the native state, three of the experimental intermediates ( $\mathrm{C} 1$ at $\Delta L=2.8 \pm 0.7$ $\mathrm{nm}, \mathrm{C} 2$ at $\Delta L=5.3 \pm 0.7 \mathrm{~nm}$ and $\mathrm{C} 3$ at $\Delta L=8.6 \pm 0.9 \mathrm{~nm}^{24}$ ) have total extensions within the range of $d$ identified for metastable species in our pulling simulations (Fig. 2a).

The emergence of intermediates can be rationalized from the changes in the folding free energy landscape upon the application of force. Using the umbrella sampling technique we have run simulations at different pulling forces and calculated 2D PMFs for the folding and extension order parameters ( $Q$ and $d$, see Fig. $2 \mathrm{~b}$ and SI for details). At low forces the dominant free energy barrier separates the unfolded well from a broad native state, with no barrier between regions with intermediate values of the order parameters and the folded state. However at $5-10 \mathrm{pN}$ a free energy barrier emerges between intermediate states that now separate from the native well. At the highest forces used in our simulations $(15-20 \mathrm{pN})$, the free energy well with $Q \simeq 0.6$ and $d \simeq 5 \mathrm{~nm}$ becomes even more stable than the native state. Hence, an external force can tilt the free energy landscape, stabilizing more extended conformations, and modulating the population of the stable forms of the protein and the barriers between them. Extended intermediates which are only transiently visited at zero force become significantly populated as the force is increased.

In order to better understand the information present in the pulling simulations and define the dominant intermediate species more systematically we integrate the data at different forces in a minimal Markovian kinetic model (i.e. an $\mathrm{MSM}^{31-33}$ ). In this type of model the dynamics of the system are encapsulated in a master equation, $d \mathbf{P}(t) / d t=\mathbf{K} \mathbf{P}(t)$, where $\mathbf{P}(t)$ is a vector with the populations of the states in the model and $\mathbf{K}$ is the rate matrix, containing the rates $k_{j i}$ for all the microscopic transitions $i \longrightarrow j$. To derive this model from the simulations we have built on established procedures, ${ }^{34,35}$ with the additional development that in this case the MSM was estimated at the different pulling forces (see full details in SI Methods). We have used a contact map-based clustering ${ }^{34,36}$ to identify a small number of states $(N=4)$, two of which are intermediates $\left(I_{1}\right.$ and $I_{2}$, see Fig. 2c) plus the 
unfolded (U) and native (N) states. On the PMFs in Fig. 2b we show the mean values of $Q$ and $d$ of the four states in the MSM. The two intermediates $\mathrm{I}_{1}$ and $\mathrm{I}_{2}$ differ in the fraction of native contacts for $\beta$-strand 1 that are broken, with only the $\mathrm{N}$-terminal residues being unfolded in $\mathrm{I}_{2}$, and the whole of strand 1 being unfolded in $\mathrm{I}_{1}$ (see contact maps in Fig. S5). The intermediate states have extensions of 2.8 and $4.8 \mathrm{~nm}$, respectively, which are close to the extensions of the experimental intermediates $\mathrm{C} 1$ and $\mathrm{C} 2,{ }^{24}$ and agree well with the locations of the free energy minima in the PMF, which were not used in the clustering process. We recolour the time series in Fig. 2a based on the MSM state to show how the assignment procedure captures the important slow dynamics (see also Fig. S4). After assigning the trajectory frames to the MSM states, we calculate the microscopic rate coefficients, $k_{j i}$ (from state $i$ to state $j$ ), at the different pulling forces using a lifetime based method. 33

The force dependence of the rate coefficients shows the expected behaviour (see Fig. 3a-b), with refolding processes being more sensitive to the pulling force than unfolding processes, due to the larger changes in molecular extension associated with refolding, and the extensible nature of the unfolded state. ${ }^{37}$ Naturally, in some cases transitions are not observed due to the finite time of the simulations. This is the case, for example, of refolding events at high pulling forces (e.g. $\mathrm{U} \longrightarrow I_{1}$ or $\mathrm{I}_{1} \longrightarrow F$ ), where the bias is too strong for the refolding process to be observed. To overcome this difficulty, we fit the calculated rates to the Bell or Dudko-Hummer-Szabo models for barrier crossing processes induced by force ${ }^{38,39}$ (in the case of $k_{\mathrm{UI}_{1}}$ and $k_{\mathrm{UI}_{2}}$ an additional intrinsic rate was also included to model the variation of folding pathway with pulling force, ${ }^{10}$ see Supplementary Methods). We find that in all cases the microscopic rate coefficients can be well captured by the functional form of the model. From the fits we can recover a rate matrix at any desired pulling force, $\mathbf{K}_{\mathrm{fit}}(F)$, even at forces where some transitions are not observed directly. Although in some cases this requires an extrapolation, it is based on the fit to a physical model.

Having assembled this model, we can attempt to recover the phenomenology observed in different conditions. We use the solution to the master equation, $\mathbf{P}(t)=\exp \left(\mathbf{K}_{\mathrm{fit}} t\right) \mathbf{P}(0)$ to propagate the dynamics and relax from a given initial condition, $\mathbf{P}(0)$. In native conditions we relax from the unfolded state, mimicking a stopped flow refolding experiment or the average behaviour of many refolding trajectories by quenching the force in the AFM. We find that the system relaxes to the equilibrium distribution with most of the amplitude (99\%) corresponding to the slowest exponential phase (see Fig. 3b, top). This slowest mode corresponds to the first eigenvector of the rate matrix $\left(\psi^{1}\right)$, which indicates exchange of density between the unfolded state and the folded and intermediate states (i.e. the folding process, see Fig. 3c). This observation is the kinetic counterpart of the dominant barrier separating the unfolded and folded wells, the latter of which here incorporates the MSM states $F, I_{1}$ and $I_{2}$ (Fig. 1b). The intermediates equilibrate rapidly with the folded state as dictated by fast processes corresponding to eigenvectors $\psi^{2}$ and $\psi^{3}$ (see Fig. 3c, bottom). In ensemble experiments, this situation, involving a single exponential phase, would inequivocally correspond to two-state folding. ${ }^{20}$ 
As we increase the force the model yields a substantially different scenario (see Fig. 3b). Propagating the rate matrix from the folded state we find that, particularly for forces $>10$ $\mathrm{pN}$ an intermediate state accumulates as the protein unfolds, reaching up to $\sim 80 \%$ of the population. When we propagate the rate matrix from the folded configuration, we find that, in a first kinetic phase, the population of the intermediates increases substantially (mostly for $\mathrm{I}_{2}$, as dictated by the eigenvector corresponding to the slowest process), and only later do they interconvert with the unfolded conformation. Again, the eigenvectors help us understand how the slowest process at higher forces involves the interconversion between $I_{1}$ and $\mathrm{U}$ (see $\psi^{1}$ in Fig. 3) while equilibration between the folded state and the intermediates is faster $\left(\psi^{2}\right.$ and $\left.\psi^{3}\right)$. This explains the long-lived formation of intermediates when unfolding upon application of a pulling force, as seen experimentally. ${ }^{24}$

A natural question is why the application of force is able to expose intermediates hidden at equilibrium, and why other perturbations such as chemical denaturants do not have a similar effect: Csp Tm has always appeared two-state in ensemble kinetics experiments using chemical denaturants to destabilize the protein. ${ }^{20} \mathrm{~A}$ possible explanation lies in the relative sensitivity of the intermediates to force or denaturant. Since the effect of denaturant is, to a first approximation, proportional to the solvent-accessible surface area $(S A S A)$ of a given state, ${ }^{40}$ its effect can be estimated from a free energy landscape projected onto ( $\left.Q, S A S A\right)$, analogous to the $(Q, d)$ projections for force (Fig. S6). The obvious difference between the two PMFs is the good correlation between $S A S A$ and $Q$ for the metastable free energy basins, compared with the situation for $d$ and $Q$. The reason for that difference is most likely that SASA is more closely related to the global degree of folding, captured by the fraction of native contacts. On the other hand, larger extensions can be sampled by intermediates via only local unfolding (as indeed evident from the structures in Fig. 2). This poor correlation between extension $d$ and $Q$ means that force can selectively stabilize intermediates which have a larger extension. For example intermediate $\mathrm{I}_{1}$ has a similar extension to the unfolded state, and so at low forces is similarly stabilized; as force increases, the softer unfolded state shifts to larger extensions and becomes more stable.

Recent efforts using highly stable atomic force microscopes are starting to resolve interesting features of energy landscapes that were not observable before, as in the case of the protein Csp $T m .{ }^{24}$ This work has resulted in an apparent discrepancy between conventional (i.e. two-state) descriptions of protein folding and more intricate descriptions where alternative paths ${ }^{10,11,19}$ and novel intermediate states ${ }^{24}$ need to be accounted for. Here we have shown how a simple coarse-grained simulation model can explain many of the experimental observations. Using a force-dependent MSM derived from the simulation data in combination with models for force-induced transitions ${ }^{38,39}$ we find that low pulling forces can stabilize intermediates. A molecular explanation for the stability of the $\mathrm{I}_{1}$ and $\mathrm{I}_{2}$ intermediates may lie in the orientation of the force-bearing beta-sheet relative to the applied force. An analysis of each of the stable states shows that while the hydrogen bonds in the force-bearing sheet are aligned with the force in the case of the native state (corresponding to an unzipping mechanism), for $\mathrm{I}_{1}$ and $\mathrm{I}_{2}$, the corresponding hydrogen bonds are closer to perpendicular to the applied force (a shearing mechanism, see Fig. S7). Since proteins whose force-bearing beta sheets are oriented perpendicular to the force (such as titin I27 or the $\mathrm{B} 1$ domain of protein $\mathrm{G}$ ) are known to be mechanically strong, this may help to explain 
the relative stability of intermediates $I_{1}$ and $I_{2}$. Consistent with this, the force-dependent unfolding rates for the two intermediates $\mathrm{I}_{1} \longrightarrow \mathrm{U}$ and $\mathrm{I}_{2} \longrightarrow \mathrm{U}$ show an initial insensitivity to the pulling force, one of the signatures of a shearing mechanism, ${ }^{10,11}$ in which the mechanism only shifts at sufficiently high force from the intrinsic unfolding pathway to that which is accelerated by pulling. As a result, describing the force dependence of these unfolding rates required the sum of a force independent intrinsic rate and a force-dependent rate from a one-dimensional (1D) rate theory to fit the simulation data, while all of the other unfolding rates (including $\mathrm{N} \longrightarrow \mathrm{I}_{1}$ and $\mathrm{N} \longrightarrow \mathrm{I}_{2}$ ) could be described by $1 \mathrm{D}$ rate theory alone. At zeo force these intermediate states may be only marginally populated and remain on the folded side of the dominant barrier, hence becoming invisible in the bulk.

We anticipate that, with further improvements in instruments, intermediates may in future be observed in force experiments on other proteins in future. Evidence of intermediates with populations that can be tuned by the combination of temperature and force has also been reported in simulations using a similar structure-based model for immunoglobulin-like ddFLN. ${ }^{41,42}$ We have shown that apparent discrepancies between the results of bulk and single-molecule experiments on mechanical unfolding of a small single-domain protein can be reconciled within the context of a coarse-grained folding model. Application of an alternative bias (force, rather than denaturant) helps to reveal more details of the energy landscape by exposing previously hidden intermediate states which are more easily perturbed by the application of a biasing force than by chemical denaturant or other perturbations.

\section{Supplementary Material}

Refer to Web version on PubMed Central for supplementary material.

\section{Acknowledgments}

D.D.S. acknowledges support from the Spanish Ministry of Economy and Competitiveness (MINECO, grant CTQ2015-65320-R) and an Ikerbasque Research Fellowship. R.B.B. was supported by the Intramural Research Program of the National Institute of Diabetes and Digestive and Kidney Diseases of the National Institutes of Health. DDS acknowledges Jörg Schönfelder, Raul Pérez-Jiménez, Victor Munoz and Frank Noé for helpful discussions.

\section{References}

1. Borgia A, Williams PM, Clarke J. Single-Molecule Studies of Protein Folding. Annu Rev Biochem. 2008; 77:101-125. [PubMed: 18412537]

2. Lu H, Isralewitz B, Krammer A, Vogel V, Schulten K. Unfolding of Titin Immunoglobulin Domains by Steered Molecular Dynamics Simulation. Biophys J. 1998; 75:662-671. [PubMed: 9675168]

3. Marszalek PE, Lu H, Li H, Carrion-Vazquez M, Oberhauser AF, Schulten K, Fernandez JM. Mechanical Unfolding Intermediates in Titin Modules. Nature. 1999; 402:100-103. [PubMed: 10573426]

4. Paci E, Karplus M. Unfolding Proteins by External Forces and Temperature: The Importance of Topology and Energetics. Proc Natl Acad Sci USA. 2000; 97:6521-6526. [PubMed: 10823892]

5. Fowler SB, Best RB, Toca-Herrera JL, Rutherford TJ, Steward A, Paci E, Karplus M, Clarke J. Mechanical Unfolding of a Titin Ig Domain: (1) Structure of Unfolding Intermediate Revealed by Combining AFM, Molecular Dynamics Simulations, NMR and Protein Engineering. J Mol Biol. 2002; 322:841-849. [PubMed: 12270718] 
6. Ng SP, Rounsevell RW, Steward A, Geierhaas CD, Williams PM, Paci E, Clarke J. Mechanical Unfolding of TNfn3: the Unfolding Pathway of a fnIII Domain Probed by Protein Engineering, AFM and MD Simulation. J Mol Biol. 2005; 350:776-789. [PubMed: 15964016]

7. Best RB, Fowler SB, Toca-Herrera JL, Clarke J. A Simple Method for Probing the Mechanical Unfolding Pathway of Proteins in Detail. Proc Natl Acad Sci USA. 2002; 99:12143-12148. [PubMed: 12218181]

8. Best RB, Fowler SB, Toca-Herrera JL, Steward A, Paci E, Clarke J. Mechanical Unfolding of a Titin Ig Domain: (2) Structure of Transition State Revealed by Combining AFM, Protein Engineering and Molecular Dynamics Simulations. J Mol Biol. 2003; 330:867-877. [PubMed: 12850153]

9. Kirmizialtin S, Huang L, Makarov DE. Topography of the Free-Energy Landscape Probed Via Mechanical Unfolding of Proteins. J Chem Phys. 2005; 122:234915. [PubMed: 16008495]

10. Best RB, Paci E, Hummer G, Dudko OK. Pulling Direction as a Reaction Coordinate for the Mechanical Unfolding of Single Molecules. J Phys Chem B. 2008; 112:5968-5976. [PubMed: 18251532]

11. Graham TGW, Best RB. Force-Induced Change in Protein Unfolding Mechanism: Discrete or Continuous Switch? J Phys Chem B. 2011; 115:1546-1561. [PubMed: 21271708]

12. Dudko OK, Graham TGW, Best RB. Locating the Folding Barrier for Single Molecules Under an External Force. Phys Rev Lett. 2011; 107:208301. [PubMed: 22181779]

13. Jackson SE. How Do Small Single-Domain Proteins Fold? Fold Des. 1998; 3:R81-R91. [PubMed: 9710577]

14. Cecconi C, Shank EA, Bustamante C, Marqusee S. Direct Observation of the Three-State Folding of a Single Protein Molecule. Science. 2005; 309:2057-2060. [PubMed: 16179479]

15. Oberhauser AF, Hansma PK, Carrion-Vazquez M, Fernandez JM. Stepwise Unfolding of Titin Under Force-Clamp Atomic Force Microscopy. Proc Natl Acad Sci USA. 2001; 98:468-472. [PubMed: 11149943]

16. Fernandez JM, Li H. Force-Clamp Spectroscopy Monitors the Folding Trajectory of a Single Protein. Science. 2004; 303:1674-1678. [PubMed: 15017000]

17. Marszalek PE, Li H, Oberhauser AF, Fernandez JM. Chair-Boat Transitions in Single Polysaccharide Molecules Observed With Force-Ramp AFM. Proc Natl Acad Sci USA. 2002; 99:4278-4283. [PubMed: 11917130]

18. Shank EA, Cecconi C, Dill JW, Marqusee S, Bustamante C. The Folding Cooperativity of a Protein is Controlled by its Chain Topology. Nature. 2010; 465:637-641. [PubMed: 20495548]

19. Guinn EJ, Jagannathan B, Marqusee S. Single-Molecule Chemo-Mechanical Unfolding Reveals Multiple Transition State Barriers in a Small Single-Domain Protein. Nat Commun. 2015; 6

20. Perl D, Welker C, Schindler T, Marahiel MA, Jaenicke R, Schmid FX. Conservation of rapid twostate folding in mesophilic, thermophilic and hyperthermophilic cold shock proteins. Nat Struct Mol Biol. 1998; 5:229-235.

21. Schuler B, Lipman EA, Eaton WA. Probing the free-energy surface for protein folding with singlemolecule fluorescence spectroscopy. Nature. 2002; 419:743-747. [PubMed: 12384704]

22. Rhoades E, Cohen M, Schuler B, Haran G. Two-State Folding Observed in Individual Protein Molecules. J Am Chem Soc. 2004; 126:14686-14687. [PubMed: 15535670]

23. Hoffmann T, Tych KM, Brockwell DJ, Dougan L. Single-Molecule Force Spectroscopy Identifies a Small Cold Shock Protein as Being Mechanically Robust. J Phys Chem B. 2013; 117:1819-1826. [PubMed: 23293964]

24. Schönfelder J, Pérez-Jiménez R, Muñoz V. A Simple Two-State Protein Unfolds Mechanically Via Multiple Heterogeneous Pathways at Single-Molecule Resolution. Nat Commun. 2016; 7:11777. [PubMed: 27248054]

25. Kremer W, Schuler B, Harrieder S, Geyer M, Gronwald W, Welker C, Jaenicke R, Kalbitzer HR. Solution NMR Structure of the Cold-Shock Protein from the Hyperthermophilic Bacterium Thermotoga Maritima. Eur J Biochem. 2001; 268:2527-2539. [PubMed: 11322871]

26. Karanicolas J, Brooks CL. The Origins of Asymmetry in the Folding Transition States of Protein L and Protein G. Protein Sci. 2002; 11:2351-2361. [PubMed: 12237457]

27. Wolynes PG, Onuchic JN, Thirumalai D. Navigating the folding routes. Science. 1995; 267:16191620. [PubMed: 7886447] 
28. Kumar S, Rosenberg JM, Bouzida D, Swendsen RH, Kollman PA. The Weighted Histogram Analysis Method for Free-Energy Calculations on Biomolecules. I. The Method. J Comput Chem. 1992; 13:1011-1021.

29. Shaknovich E, Farztdinov G, Gutin AM, Karplus M. Protein folding bottlenecks: a lattice Monte Carlo simulation. Phys Rev Lett. 1991; 67:1665-1668. [PubMed: 10044213]

30. O'Brien EP, Ziv G, Haran G, Brooks BR, Thirumalai D. Effects of Denaturants and Osmolytes on Proteins Are Accurately Predicted by the Molecular Transfer Model. Proc Natl Acad Sci USA. 2008; 105:13403-13408. [PubMed: 18757747]

31. Noe F, Horenko I, Schutte C, Smith JC. Hierarchical Analysis of Conformational Dynamics in Biomolecules: Transition Networks of Metastable States. J Chem Phys. 2007; 126:155102. [PubMed: 17461666]

32. Chodera JD, Singhal N, Pande VS, Dill KA, Swope WC. Automatic Discovery of Metastable States for the Construction of Markov Models of Macromolecular Conformational Dynamics. J Chem Phys. 2007; 126:155101. [PubMed: 17461665]

33. Buchete N-V, Hummer G. Coarse Master Equations for Peptide Folding Dynamics. J Phys Chem B. 2008; 112:6057-6069. [PubMed: 18232681]

34. Carter JW, Baker CM, Best RB, De Sancho D. Engineering Folding Dynamics from Two-State to Downhill: Application to $\lambda$-Repressor. J Phys Chem B. 2013; 117:13435-13443. [PubMed: 24079652]

35. Naganathan AN, De Sancho D. Bridging Experiments and Native-Centric Simulations of a Downhill Folding Protein. J Phys Chem B. 2015; 119:14925-14933. [PubMed: 26524123]

36. Kellogg EH, Lange OF, Baker D. Evaluation and Optimization of Discrete State Models of Protein Folding. J Phys Chem B. 2012; 116:11405-11413. [PubMed: 22958200]

37. Best RB, Hummer G. Protein Folding Kinetics Under Force from Molecular Simulation. J Am Chem Soc. 2008; 130:3706-3707. [PubMed: 18307341]

38. Bell GI. Models of the specific adhesion of cells to cells. Science. 1978; 200:618-627. [PubMed: 347575]

39. Dudko OK, Hummer G, Szabo A. Intrinsic Rates and Activation Free Energies from SingleMolecule Pulling Experiments. Phys Rev Lett. 2006; 96:108101-108104. [PubMed: 16605793]

40. Myers J, Pace C, Scholtz J. Denaturant m-Values and Heat Capacity Changes: Relation to Changes in Accessible Surface Areas of Protein Unfolding. Protein Sci. 1995; 4:2138-48. [PubMed: 8535251]

41. Yew ZT, Krivov S, Paci E. Free Energy Landscapes of Proteins in the Presence and Absence of Force. J Phys Chem B. 2008; 112:16902-16907. [PubMed: 19053698]

42. Sun L, Noel JK, Sulkowska JI, Levine H, Onuchic J. Connecting Thermal and Mechanical Protein (Un)folding Landscapes. Biophys J. 2014; 107:2950-2961. [PubMed: 25517160] 

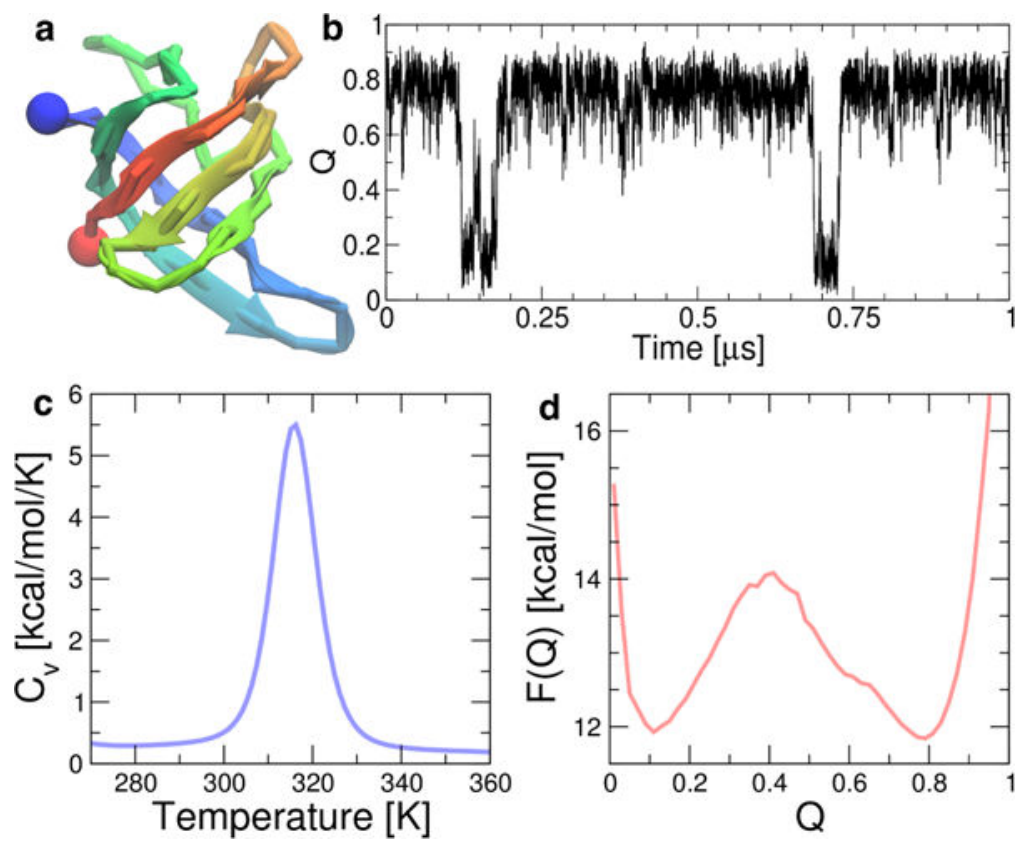

Figure 1.

(a) Cartoon representation of the cold shock protein from Thermotoga maritima (PDB: $1 \mathrm{~g} 6 \mathrm{p})$. The $\mathrm{C}^{\alpha}$ trace of the protein is shown to emphasize the resolution of the simulation model. The $\mathrm{C}$ and $\mathrm{N}$ termini of the protein from which we pull are shown as spheres. (b) Time series data corresponding to the projection on the fraction of native contacts $(Q)$ at zero force and $310 \mathrm{~K}$. (c) Heat capacity thermogram obtained from equilibrium runs at multiple temperatures. (d) Potential of mean force at the midpoint temperature. 

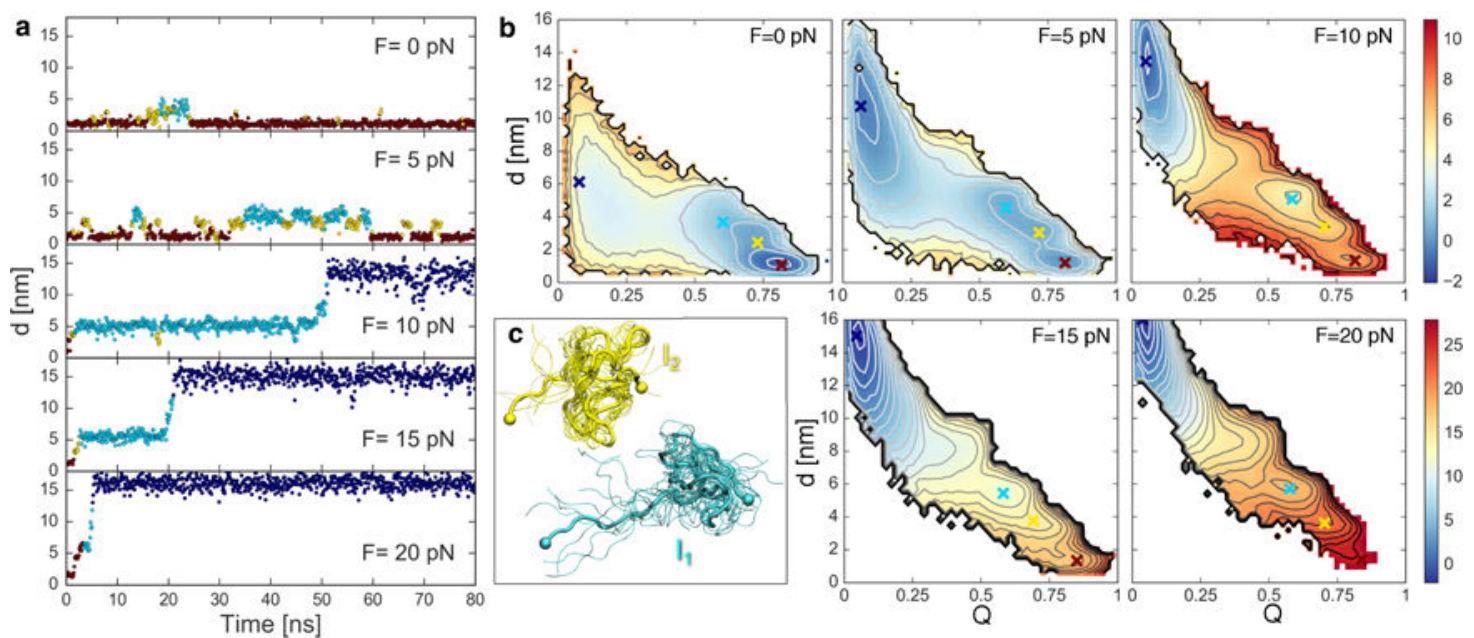

Figure 2.

(a) Projection of simulation trajectories at different pulling forces on the end to end distance, d. Data points are coloured based on the MSM state they are assigned to ( $\mathrm{U}$ : dark blue; $\mathrm{I}_{1}$ : cyan; $\mathrm{I}_{2}$ : yellow; F: brown). (b) Potentials of mean force from umbrella sampling calculated at forces between $0 \mathrm{pN}$ and $20 \mathrm{pN}$ using the fraction of native contacts $Q$ and the end to end distance $d$ as order parameters. Free energies are in $\mathrm{kcal} / \mathrm{mol}$. Note that due to the changes in stability of the states induced by force different free energy scales are used for 0,5 and 10 $\mathrm{pN}$ (top) and 15 to $20 \mathrm{pN}$ (bottom). We show the mean values of $Q$ and $d$ for MSM states calculated from snapshots assigned at the corresponding pulling forces. (c) Representative snapshots of the MSM intermediates $\mathrm{I}_{1}$ and $\mathrm{I}_{2}$. 

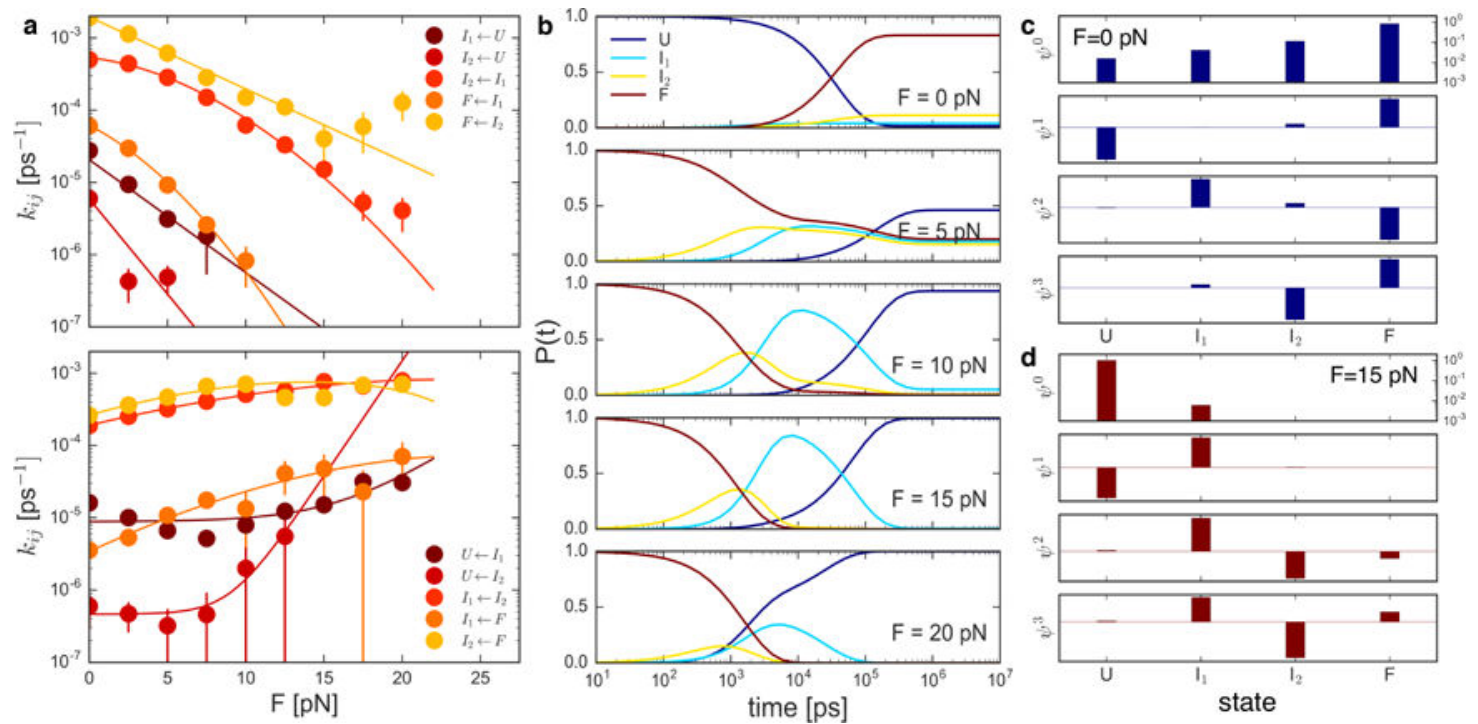

Figure 3.

(a) Microscopic rate coefficients for transitions in the refolding (top) and unfolding (bottom) directions. Lines are fits to the one dimensional barrier model (see text). (b) Relaxation to equilibrium of the population of different states $P(t)$ at different forces. (c, d) Eigenvectors of the fitted rate matrix at 0 and $15 \mathrm{pN}$, respectively.

J Phys Chem Lett. Author manuscript; available in PMC 2017 October 06. 